\title{
Biomechanical and biochemical outcomes of porcine temporomandibular joint disc
} deformation

\author{
Andrea M. Matuska ${ }^{1}$, Stephen Muller ${ }^{1}$, M. Franklin Dolwick ${ }^{2}$, Peter S. McFetridge ${ }^{1 *}$ \\ ${ }^{1}$ J. Crayton Pruitt Family Department of Biomedical Engineering, University of Florida \\ ${ }^{2}$ Department of Oral and Maxillofacial Surgery, University of Florida
}




\section{ABSTRACT}

Objective: The structure-function relationship in the healthy temporomandibular joint (TMJ) disc has been well established, however the changes in dysfunctional joints has yet to be systematically evaluated. Due to the poor understanding of the etiology of temporomandibular disorders (TMDs) this study evaluated naturally occurring degenerative remodeling in aged female porcine temporomandibular joint (TMJ) discs in order to gain insight into the progression and effects on possible treatment strategies of TMDs. Design: Surface and regional biomechanical and biochemical properties of discal tissues were determined in grossly deformed ( $\geq$ Wilkes Stage 3) and morphologically normal ( $\leq$ Wilkes Stage 2) TMJ discs. Results:

Compared to normal disc structure the deformed discs lacked a smooth biconcave shape and characteristic ECM organization. Reduction in tensile biomechanical integrity and increased compressive stiffness and cellularity was found in deformed discs. Regionally, the posterior and intermediate zones of the disc were most frequently affected along with the inferior surface. Conclusions: The frequency of degeneration observed on the inferior surface of the disc (predominantly posterior), suggests that a disruption in the disc-condyle relationship likely contributes to the progression of joint dysfunction more than the temporodiscal relationship. As such, the inferior joint space may be an important consideration in early clinical diagnosis and treatment of TMDs, as it is overlooked in techniques performed in the upper joint space, including arthroscopy and arthrocentesis. Furthermore, permanent damage to the disc mechanical properties would limit the ability to successfully reposition deformed discs, highlighting the importance of emerging therapies such as tissue engineering. Keywords: Bioengineering, Extracellular matrix (ECM), Functional morphology, Biomechanics, Remodeling, Temporomandibular disorders (TMD) 


\section{INTRODUCTION}

The temporomandibular joint (TMJ) is a mechanically and biologically complex joint comprising of a thick fibrocartilage disc that minimizes the incongruity between the articulation of the fossa-eminence of the temporal bone and mandibular condyle. Normally, the biconcave disc dynamically adapts to distribute compressive, tensile and shear stress throughout a sequence of movements (1-3). The inferior surface of the disc primarily experiences rotation through its close association to the condyle while the superior surface experiences mainly translation along the temporal bone $(4,5)$.

Temporomandibular disorders (TMDs) are a wide range of disorders that affect both the joint and/or the associated structures; however the TMJ disc is affected in as many as $70 \%$ of all cases (6-8). Patients are usually affected by unidirectional or multidirectional displacement of the intra-articular disc either anteriorly, medially, laterally, or (very rarely) posteriorly $(9,10)$. In disc displacement, the disc can either be recaptured into a normal position during mouth opening termed disc displacement with reduction (DDWR), or can remain displaced termed disc displacement without reduction (DDWOR). An MRI study by Hirata et al showed disc morphology to remain primarily biconcave (79\%) in DDWR, while disc morphology is altered in DDWOR. Disc shape transitions to a predominantly folded configuration (43\%) in addition to hemiconvex (21\%) and biconvex (21\%) shapes DDWOR (11). The frequently used Wilkes scale groups TMDs into five stages of increasing dysfunction, with Stage III indicative of DDWOR, resulting in obvious disc deformation, which disrupts the discs ability to adapt to the condyle and fossa $(9,12)$.

The complex loading normally distributed by the disc leads to a very distinct regional variations and structure-function relationships in a healthy joint (13). It would be expected that 
the disruption of normal function in dysfunctional joints would results in adaptive remodeling and degenerative changes to the disc structure that could be systematically evaluated. Similarity between porcine and human TMJ anatomy has led to many bioengineering studies utilizing the porcine TMJ disc as a model. Similarities include size, extracellular matrix alignment, collagen content, region specific biomechanical and biochemical properties, and an omnivorous diet leading to similar mechanical loading conditions (14-18).

Using naturally deformed female porcine discs as a model structure, this study sought to determine how surface and regional biomechanical and biochemical properties of discal tissues differ between grossly deformed and morphologically normal TMJ discs from the same population. The hypothesis was that by studying how the structure of the disc is altered in deformation, understanding of the functional changes of the diseased joint can be elucidated which may provide insight into the identification, progression, and influence of treatment strategies of TMDs.

\section{MATERIALS \& METHODS}

Tissue collection

Unmatched en bloc porcine temporomandibular joints were obtained from Yorkshire sows (IACUC \# 201207534, Animal Technologies, Tyler, TX, n=12; 7 left/5 right). Exact age was unknown, however they were known to be retired breeding sows aged $2+$ years. Discs were dissected from the joints, with peripheral attachments left intact, by disarticulating the joint after severing the masseter and lateral pterygoid. The criterion for disc deformation was the lack of smooth biconcavity after removal from the joint correlating with progression to Wilkes stage 3. Following dissection of the disc and washing in phosphate-buffered saline (PBS), they were 
stored frozen at $-20^{\circ} \mathrm{C}$ in gauze soaked with saline containing protease inhibitors $(2.3 \mathrm{mM}$ EDTA [Acros Organics, Morris Plains, NJ], $10 \mathrm{mM}$ N-ethylmaleimide, and $1 \mathrm{mM}$ phenylmethanesulfonyl fluoride [Sigma, St. Louis, MO, USA (17)]).

\section{Sample Preparation}

On the day of testing, discs were thawed and equilibrated in PBS at $37^{\circ} \mathrm{C}$ for 1 hour. Before regional specimen isolation, disc measurements were obtained in triplicate using calipers in the anterior-posterior (AP) and medial-lateral (ML) direction as well as the thickness of the posterior band (PB), intermediate zone (IZ), and anterior band (AB). Duplicate tensile specimens in the lateral, central, and medial portions of the disc were prepared using a custom parallel blade apparatus to produce $1 \mathrm{~mm}$ wide section in the anterior-posterior direction and then cut to $10 \mathrm{~mm}$ in length. Lastly, superior and inferior surface samples were obtained by sectioning $1 \mathrm{~mm}$ sections from the superior and inferior surfaces after the desired surface was pressed flat against another surface. Posterior and anterior tensile specimens were prepared partly into the attachment tissues to give a measure of disc/attachment strength. Compressive specimens were prepared using $3 \mathrm{~mm}$ biopsy punches (Miltex, York, PA) in the lateral, medial, anterior, posterior and intermediate portions of the disc. The top and bottom $1 \mathrm{~mm}$ of the punches were obtained for superior and inferior testing samples as were obtained in tensile testing (Figure 1).

\section{Biomechanical Evaluation}

The ends of the rectangular tensile specimens were mounted vertically between two hemostats, leaving a $5 \mathrm{~mm}$ gauge length. An Instron 5542 mechanical testing instrument (Instron Corporation; Norwood, MA, USA) was used to apply an extension rate of $1 \%$ strain/sec (19) within a PBS hydrated chamber at room temperature following cyclic preconditioning in the toe region at 5\% strain (1). Young's modulus $(E)$, ultimate tensile strength (UTS), and strain at 
failure were calculated from stress-strain curves. Samples that broke at the grips were discarded from analysis and where duplicate measures were obtained they were averaged together before further analysis.

Compressive disc specimens were placed in a hydrated testing chamber in a Biomomentum Mach-1 micromechanical system (Biomomentum Inc, Laval, Quebec, Canada) and subject to cyclic compression tests consisting of 15 cycles at $5 \%$ strain performed at $0.5 \mathrm{~Hz}$. Settings were programed after an initial find contact with $0.05 \mathrm{~N}$. Steady state compressive modulus was determined from the slope of the average loading curve of the last five cycles (3).

\section{Biochemical Evaluation}

Following compressive biomechanical testing, discs were weighed and digested in papain buffer $(1.5 \mathrm{mg} / \mathrm{ml}$ papain [Spectrum Chemical, Gardena CA], 1mM EDTA [Acros Organics], 5.5 mM N-acetylcysteine [Thermo Scientific, Rockford, IL], 0.067 mM $\beta$-mercaptoethanol [Sigma Aldrich] in PBS) for 20 hours at $60^{\circ} \mathrm{C}$. DNA quantification of the digest was performed using the Quant-IT Picogreen dsDNA assay (Life Technologies, Grand Island, NY) and DNA was converted to cell number using a conversion of 7.7pg DNA/cell (20) and divided by the volume of the tissue to give a cell density. Sulfated glycosaminoglycan (sGAG) content in the digests were determined using a DMMB colorimetric assay (21) and normalized to the wet weight of the tissue.

Histology

Cellularity and extracellular matrix alignment in the sagittal and transverse anatomical planes were visualized with remaining untested tissue (Figure 1). Tissues were embedded in Neg50 media (Richard Allen Scientific) and $10 \mu \mathrm{m}$ sections were obtained with a HM 550 
cryostat (Thermo Scientific). Sections were stained with standard hematoxylin \& eosin, mounted, and images were acquired with an Axiocam ICc. 1 at 20x objective magnification. Statistics

Mean and standard deviation were determined for all samples. A two-tailed two-sample ttest was used to determine significant differences between normal $(n=7)$ and deformed $(n=4)$ tissue gross measurements $(\alpha=0.05)$. Three-factor full factorial ANOVAs were performed on biomechanical and biochemical outcomes to evaluate the main and interaction effects between regional, surface, and deformation factors. When significance was indicated, post-hoc Fisher's LSD with a Bonferroni correction was performed. Corrected significance was set at $\alpha=0.005$ for regional differences, $\alpha=0.01$ for deformation/regional and surface/regional interactions, and $\alpha=0.025$ for surface/deformation interactions.

\section{RESULTS}

\section{Overall Morphology}

Of the 12 discs isolated, the overall incidence of disc deformation was found to be $42 \%$ ( $\mathrm{n}=7$ normal, $\mathrm{n}=5$ deformed), with no identifiable perforations. Macroscopic examination showed a folded (flexure) deformation in four of the deformed discs while one disc was hemiconvex (Figure 2A). Since a folded disc configuration is seen more commonly than hemi-convex configuration, and likely occurs via a different mechanism, the hemi-convex disc was excluded from the statistical analysis to better identify significant factors $(11,22)$. Normal $(n=7)$ and deformed $(\mathrm{n}=4)$ discs did not vary significantly in medial-lateral $(\mathrm{p}=0.100)$ and anterior-posterior $(\mathrm{p}=0.132)$ length (Figure 2B). Statistically significant thickening of the posterior $(\mathrm{p}=0.015)$, intermediate $(\mathrm{p}=0.007)$, and anterior $(\mathrm{p}=0.005)$ zones of the deformed discs was found compared to morphologically normal discs (Figure 2C). 


\section{Tensile Biomechanical Evaluation}

Young's Modulus and ultimate tensile strength (UTS) were significantly affected by deformation $(\mathrm{p}<0.001, \mathrm{p}<0.001)$, surface $(\mathrm{p}=0.020, \mathrm{p}=0.023)$, regional $(\mathrm{p}<0.001, \mathrm{p}<0.001)$ factors as well as deformation-region $(\mathrm{p}<0.001, \mathrm{p}=0.007)$ and deformation-surface ([UTS only] $\mathrm{p}=0.058, \mathrm{p}=0.049$ ) interactions (Figure 3A, B).

Deformed tissues had significantly lower tensile modulus and UTS than morphologically normal tissues. In morphologically normal discs, average Young's Modulus and UTS was 0.84.7 and 1.1-3.2 times that of corresponding values in deformed tissues, respectively. Overall, inferior surfaces had significantly higher modulus and UTS values than superior surfaces. Posthoc analysis of the deformation-surface interaction showed deformation resulted in a more pronounced reduction of UTS on the inferior surface $(\mathrm{p}<0.0001)$ than on the superior surface $(\mathrm{p}=0.01)$.

Regionally, the tensile modulus and UTS in the intermediate zone was significantly higher than in all other regions $(\mathrm{p}<0.0001)$. The values in the medial region were also significantly higher than in the anterior $(\mathrm{p}<0.0001)$ and posterior $(\mathrm{p}<0.0003)$ regions. Only the modulus in the medial region was near significantly greater than the lateral region $(\mathrm{p}=0.006)$. Interaction between deformation and region factors showed a significant interaction effect on UTS and modulus between deformation and the intermediate zone $(\mathrm{p}<0.0001)$.

Strain at failure was significantly affected by deformation $(\mathrm{p}=0.019)$ and region $(p<0.001$, Figure 3C). Deformed tissues had greater elongation at failure than normal tissues. Regionally, the posterior region had significantly greater elongation than medial, intermediate zone, and lateral regions $(\mathrm{p}<0.0001)$. 


\section{Compressive Biomechanical Evaluation}

Compressive modulus was significantly affected by deformation $(\mathrm{p}=0.005)$ and regional $(\mathrm{p}=0.024)$ factors as well as deformation-region $(\mathrm{p}=0.016)$ and surface-regional $(\mathrm{p}=0.028)$ interactions (Figure 4). Deformed disc specimens were stiffer overall in compression than normal discs. Even though the ANOVA showed significance, corrected post-hoc evaluation of regional differences did not identify any significant differences, though posterior region was near significant $(\mathrm{p}=0.006)$. The interaction between the region and deformation, however, was significant in the posterior region $(\mathrm{p}=0.002)$. The compressive modulus in the posterior region of deformed discs was 1.95-2 times higher than in morphologically normal discs. Surface and regional interactions while significant in the ANOVA showed no significance in corrected posthoc values, only near significance between inferior and superior surfaces in posterior region $(\mathrm{p}=0.016)$.

\section{Quantification of Cell Density and sGAG Content}

Cell density was significantly affected by deformation $(\mathrm{p}<0.001)$ and regional $(\mathrm{p}<0.001)$ factors (Figure 5A). Deformed discs had significantly higher cell density than morphologically normal discs. The lateral region of the discs had significantly higher cell density than medial $(\mathrm{p}=0.0005)$ and $\mathrm{IZ}(\mathrm{p}=0.0001)$ regions. Only regional factors were significant in regards to sGAG concentration $(\mathrm{p}<0.001$, Figure $5 \mathrm{~B})$. Intermediate and medial regions had the highest sGAG content, followed by lateral region, posterior and anterior bands. A summary of all statistical results are shown in Table 1.

\section{Histology}

H\&E staining in the intermediate zone of the normal and deformed discs showed lack of characteristic anterior-posterior fiber alignment and clusters of cells in the deformed discs. There 
were also more round shaped cells compared to spindle shaped cells (Figure 6). Cell dense nodules were also observed on the posterior inferior surface of 3/4 deformed discs (Figure 7).

\section{DISCUSSION}

A high incidence of deformation was observed in this study (42\%) compared to estimates that TMDs affect $25-30 \%$ of the human population (23). These estimates relate to symptomatic clinical findings which are not always associated with anatomical findings (24). Over half the population over 50 shows radiographic evidence of degenerative changes in their joints with this number increasing to $85 \%$ by age 75 (25). Furthermore, the discs used in this study were acquired from a retired female breeder population and it is well known that TMJ disorders predominantly affect women potentially due to hormonal differences (26). Therefore such a high occurrence of deformation is attributed to the choice of sample population.

Macroscopically, deformed porcine discs, which lacked smooth biconcavity, displayed many similarities to deformed human discs such as thickening of the posterior band and intermediate zone $(27,28)$. The predominance of a folded disc configuration observed here mirrors results from an MRI study showing a folded type deformation was the predominant (43\%) configuration of discs displaced without reduction (11). Due to the advanced age of the pigs, all dimensions of the discs measured in this study were larger than similar measurements reported in literature $(14,29)$. The majority of other comparative studies use discs obtained from animals aged 3-12 months $(14,19,20,30,31)$ however skeletal maturity is not reached until at least 1 year of age (15).

Gross remodeling was noticeable on both the superior and inferior surfaces of the disc, with the majority of deformed discs demonstrating remodeling consistent with what appears to be an upward flexure in the intermediate zone. In an MRI study of human TMJ discs of patients 
with anterior disc displacement, upward flexure was less common than downward flexure but more often associated with advanced clinical symptoms and non-reducing disc displacement that would result in permanent deformation (32).

The inferior and superior surfaces of the TMJ disc separate the joint into separate upper and lower joint spaces that experience distinct mechanical forces (33), thus necessitating biomechanical characterization of the surfaces independently. In deformed discs, tensile mechanical properties were significantly lower in deformed discs compared to morphologically normal discs. This effect was most pronounced on the inferior surface as well as in the entire intermediate zone. However, in compression, deformed discs were generally stiffer, with posterior region most affected. These outcomes are directly supported by a finite element study performed by Tanaka et al. where it was found that displaced discs experienced increased compressive forces in posterior and medial tissues as well as a reduction in normal tensile forces (34).

A structure-function relationship exists between the direction of mechanical forces experienced by the disc and the organization of the extracellular matrix (1). As demonstrated in this study the intermediate zone of the morphologically normal disc has a tensile Young's modulus up to $150 \mathrm{MPa}$ in in the anterior-posterior direction which correlated with a strong anterior-posterior collagen fiber alignment in the ECM. However, the tensile Young's modulus in deformed discs was less than $70 \mathrm{MPa}$, and reorganization of the ECM was evident. In terms of clinical treatment, once the disc has deformed, recontouring and repositioning may no longer be effective as the disc cannot withstand physiologic loading forces. This is supported by the results of a retrospective study in which it was found that patients with disc deformation (Wilkes stage 
III, or higher) experienced more frequent rupture and possible rupture of the intermediate zone of the disc following repositioning (35).

A number of studies have investigated the tensile properties of healthy TMJ discs and all have employed varying testing protocols. The results of this study followed the same trend as what has been observed in other studies where the intermediate zone has the highest tensile modulus than other discal regions tested in the same (A-P) direction, followed by the medial and lateral regions $(1,36)$. While this is the first study to measure the superior and inferior regions independently, the modulus of morphologically normal discs measured in this study was slightly higher what has been measured in previous studies (149 MPa in the inferior region and $124 \mathrm{MPa}$ in the superior region). Studies by Beatty et al and Tanne et al $(36,37)$ report overall tensile Young's modulus values at 76.4 and $101.1 \mathrm{MPa}$, respectively. The age of the discs may have played a role in the higher modulus values. Tanaka et al has shown age related changes to the TMJ disc result in increased elastic tensile stiffness (38).

GAG content has been associated with the TMJ discs' compressive properties (39). While trending toward an increase in sGAG content in deformed tissues, the lack of significant variation in sGAG content between normal and deformed discs indicates the higher compressive stiffness observed in the deformed discs is likely a result of collagen reorganization and/or fibrosis rather than sGAG deposition. Permanent deformation is likely secondary to displacement (22), therefore, ECM reorganization is in response to altered mechanical loading. Following similar testing methods sGAG content in the healthy porcine disc has been reported between $0.31-0.43 \%$ per wet weight as well as $0.96 \pm 0.39 \%$ per dry weight of tissue $(14,20)$. Similarly, in this study sGAG content ranged from $0.26-0.59 \%$ per wet weight of the tissue. 
The inferior surface of the disc has a close association with the condyle and the surrounding attachments are responsible for stabilization of the discs position on condyle. Therefore, the mechanical integrity of the posterior attachment to the condyle may play an important role in the progression of displacement. This is supported by Eriksson et al showing displacement can be induced with superficial incisions to that region (40) as well as clinical findings where perforations are noted predominantly in the inferior posterior attachments (41, 42). A biomechanical study by Murphy et al showed that high strains in porcine TMJ disc posterior attachments were observed in the anterioposterior direction, leading to the conclusion that compromise of these attachments may contribute to displacement (19). This results of this study confirmed that the inferior posterior tissue of deformed discs was significantly weaker than that of morphologically normal tissue in anterioposterior tensile tests.

Another interesting phenomenon was localized cellular hyperplasia in the posterior inferior region of the TMJ discs studied. This hyperplastic response may further obstruct rotational movement, through increased thickness and friction between the articulation of the disc and condyle, and induce an inflammatory response contributing to degeneration in the discal tissues and potential advancement of disc displacement. One potential explanation for this observed response is microtrauma due to overextension in the posterior region resulting in localized cell proliferation as a wound response. Hyperplasia on the inferior surface of the disc has been seen in human discs as well. Kirk examined discs removed from symptomatic patients and also found hyperplasia on the inferior surface which he associated with interference to condylar movement (43).

As to be expected, cell density determined from DNA measurements of the discs indicated a higher cell density than what is reported in other studies. In healthy, young discs, cell 
number has been described as $21-83 \times 10^{6}$ cells $/ \mathrm{ml}$ and $50 \times 10^{6}$ cells $/ \mathrm{g}(20,29)$. In aged, but morphologically normal discs cell density ranged from $93-173 \times 10^{6}$ cells $/ \mathrm{cm}^{3}$. However in deformed discs, cell density ranged from $111-276 \times 10^{6}$ cells $/ \mathrm{cm}^{3}$.

Limitations of this study include the assumption of advanced displacement based on deformed disc morphology. Furthermore, normal disc morphologically does not guarantee absence of disc displacement (24) and findings from morphologically normal discs should not be considered as results from healthy discs. Symptomatic clinical findings are not always associated with anatomical findings, and we have no indication whether there was any pain associated with the discs. Lastly, discussion of results are compared to human disc displacement, however differences between human and porcine TMJ disc exist. Human retrodiscal tissues are highly vascular, whereas porcine retrodiscal tissue is more fibrofatty (44). A difference in mastication frequency has also been established, though the effect on disc displacement or disease is unknown.

In conclusion, the results of this study highlight the significance of the inferior surface of the disc in progression in disorders of the temporomandibular joint disc. This is particularly relevant to current techniques, arthroscopy and arthrocentesis, that are most often performed in the upper joint space. Furthermore, the resulting compromise in disc mechanical properties, especially in the intermediate zone limits clinical treatments available for patients presenting painful pathologic disc morphology emphasizing the importance of early diagnosis and early intervention (prior to disc deformation) to improve clinical outcomes. Continued efforts in regenerative medicine, such as tissue engineering (which has mostly focused on the intermediate zone), have the potential to offer a viable treatment alternative once disc deformation has occurred. 


\section{ACKNOWLEDGEMENTS}

We would like to thank Emily Lakes for input in performing biomechanical tests. We gratefully acknowledge the support of the National Institute of Dental and Craniofacial Research at the US National Institutes of Health (NIH; 1R21DE022449) and the National Science Foundation Graduate Research Fellowship (DGE-1315138). Any opinion, findings, and conclusions or recommendations expressed in this material are those of the authors and do not necessarily reflect the views of the National Institutes of Health or National Science Foundation. The authors declare no potential conflicts of interest in respect to the authorship and/or publication of this article. 


\section{REFERENCES}

1. Detamore MS, Athanasiou KA. Tensile properties of the porcine temporomandibular joint disc. J Biomech Eng 2003;125(4):558-565.

2. Allen KD, Athanasiou KA. Viscoelastic characterization of the porcine temporomandibular joint disc under unconfined compression. J Biomech 2006;39(2):312-322.

3. Juran CM, Dolwick MF, McFetridge PS. Shear mechanics of the TMJ disc: relationship to common clinical observations. J Dent Res 2013;92(2):193-198.

4. Griffin CJ, Hawthorn R, Harris R. Anatomy and histology of the human temporomandibular joint. Monogr Oral Sci 1975;4:1-26.

5. Piette E. Anatomy of the human temporomandibular joint. An updated comprehensive review. Acta Stomatol Belg 1993;90(2):103-127.

6. Farrar WB, McCarty WL, Jr. Inferior joint space arthrography and characteristics of condylar paths in internal derangements of the TMJ. J Prosthet Dent 1979;41(5):548-555.

7. Schiffman EL, Truelove EL, Ohrbach R, Anderson GC, John MT, List T, et al. The Research Diagnostic Criteria for Temporomandibular Disorders. I: overview and methodology for assessment of validity. J Orofac Pain 2010;24(1):7-24.

8. Ahmad M, Hollender L, Anderson Q, Kartha K, Ohrbach R, Truelove EL, et al. Research diagnostic criteria for temporomandibular disorders (RDC/TMD): development of image analysis criteria and examiner reliability for image analysis. Oral Surg Oral Med Oral Pathol Oral Radiol Endod 2009;107(6):844-860.

9. Molinari F, Manicone PF, Raffaelli L, Raffaelli R, Pirronti T, Bonomo L.

Temporomandibular joint soft-tissue pathology, I: Disc abnormalities. Semin Ultrasound CT MR 2007;28(3):192-204.

10. Farrar WB, McCarty WL, Jr. The TMJ dilemma. J Ala Dent Assoc 1979;63(1):19-26.

11. Hirata FH, Guimaraes AS, Oliveira JX, Moreira CR, Ferreira ET, Cavalcanti MG.

Evaluation of TMJ articular eminence morphology and disc patterns in patients with disc displacement in MRI. Braz Oral Res 2007;21(3):265-271.

12. Wilkes CH. Internal derangements of the temporomandibular joint. Pathological variations. Arch Otolaryngol Head Neck Surg 1989;115(4):469-477.

13. Detamore MS, Athanasiou KA. Structure and function of the temporomandibular joint disc: implications for tissue engineering. J Oral Maxillofac Surg. United States: 2003 American Association of Oral and Maxillofacial Surgeons J Oral Maxillofac Surg 61:494-506, 2003, 2003. p. 494-506.

14. Kalpakci KN, Willard VP, Wong ME, Athanasiou KA. An interspecies comparison of the temporomandibular joint disc. J Dent Res 2011;90(2):193-198.

15. Herring SW, Decker JD, Liu ZJ, Ma T. Temporomandibular joint in miniature pigs: anatomy, cell replication, and relation to loading. Anat Rec 2002;266(3):152-166.

16. Herring SW. TMJ anatomy and animal models. J Musculoskelet Neuronal Interact 2003;3(4):391-394; discussion 406-397.

17. Allen KD, Athanasiou KA. A surface-regional and freeze-thaw characterization of the porcine temporomandibular joint disc. Ann Biomed Eng 2005;33(7):951-962.

18. Detamore MS, Orfanos JG, Almarza AJ, French MM, Wong ME, Athanasiou KA.

Quantitative analysis and comparative regional investigation of the extracellular matrix of the porcine temporomandibular joint disc. Matrix Biol 2005;24(1):45-57.

19. Murphy MK, Arzi B, Hu JC, Athanasiou KA. Tensile characterization of porcine temporomandibular joint disc attachments. J Dent Res 2013;92(8):753-758. 
20. Almarza AJ, Bean AC, Baggett LS, Athanasiou KA. Biochemical analysis of the porcine temporomandibular joint disc. Br J Oral Maxillofac Surg 2006;44(2):124-128.

21. Mort JS, Roughley PJ. Measurement of glycosaminoglycan release from cartilage explants. Methods Mol Med 2007;135:201-209.

22. Murakami S, Takahashi A, Nishiyama H, Fujishita M, Fuchihata H. Magnetic resonance evaluation of the temporomandibular joint disc position and configuration. Dentomaxillofac Radiol 1993;22(4):205-207.

23. Solberg WK, Woo MW, Houston JB. Prevalence of mandibular dysfunction in young adults. J Am Dent Assoc 1979;98(1):25-34.

24. Westesson PL, Eriksson L, Kurita K. Reliability of a negative clinical temporomandibular joint examination: prevalence of disk displacement in asymptomatic temporomandibular joints. Oral Surg Oral Med Oral Pathol 1989;68(5):551-554.

25. Haskin CL, Milam SB, Cameron IL. Pathogenesis of degenerative joint disease in the human temporomandibular joint. Crit Rev Oral Biol Med 1995;6(3):248-277.

26. Warren MP, Fried JL. Temporomandibular disorders and hormones in women. Cells Tissues Organs 2001;169(3):187-192.

27. Mills DK, Daniel JC, Herzog S, Scapino RP. An animal model for studying mechanisms in human temporomandibular joint disc derangement. J Oral Maxillofac Surg 1994;52(12):1279-1292.

28. Kurita K, Westesson PL, Sternby NH, Eriksson L, Carlsson LE, Lundh H, et al. Histologic features of the temporomandibular joint disk and posterior disk attachment: comparison of symptom-free persons with normally positioned disks and patients with internal derangement. Oral Surg Oral Med Oral Pathol 1989;67(6):635-643.

29. Kuo J, Shi C, Cisewski S, Zhang L, Kern MJ, Yao H. Regional cell density distribution and oxygen consumption rates in porcine TMJ discs: an explant study. Osteoarthritis Cartilage 2011;19(7):911-918.

30. Detamore MS, Hegde JN, Wagle RR, Almarza AJ, Montufar-Solis D, Duke PJ, et al. Cell type and distribution in the porcine temporomandibular joint disc. J Oral Maxillofac Surg. United States, 2006. p. 243-248.

31. Willard VP, Arzi B, Athanasiou KA. The attachments of the temporomandibular joint disc: a biochemical and histological investigation. Arch Oral Biol 2012;57(6):599-606.

32. Yoshida H, Hirohata H, Onizawa K, Niitsu M, Itai Y. Flexure deformation of the temporomandibular joint disk in pseudodynamic magnetic resonance images. Oral Surg Oral Med Oral Pathol Oral Radiol Endod 2000;89(1):104-111.

33. Bermejo-Fenoll A, Panchon-Ruiz A, Gonzalez-Gonzalez JM, Gonzalez Sequeros O. A study of the movements of the human temporomandibular joint complex in the cadaver. Cranio 2002;20(3):181-191.

34. Tanaka E, Rodrigo DP, Miyawaki Y, Lee K, Yamaguchi K, Tanne K. Stress distribution in the temporomandibular joint affected by anterior disc displacement: a three-dimensional analytic approach with the finite-element method. J Oral Rehabil 2000;27(9):754-759.

35. Li H, Cai X, Yang C, Wang S, Huang L. Ruptured disc after arthroscopic repositioning in the temporomandibular joint: a retrospective magnetic resonance imaging study. $\mathrm{Br}$ J Oral Maxillofac Surg 2014;52(6):545-550.

36. Tanne K, Tanaka E, Sakuda M. The elastic modulus of the temporomandibular joint disc from adult dogs. J Dent Res 1991;70(12):1545-1548. 
37. Beatty MW, Bruno MJ, Iwasaki LR, Nickel JC. Strain rate dependent orthotropic properties of pristine and impulsively loaded porcine temporomandibular joint disk. J Biomed Mater Res 2001;57(1):25-34.

38. Tanaka E, Sasaki A, Tahmina K, Yamaguchi K, Mori Y, Tanne K. Mechanical properties of human articular disk and its influence on TMJ loading studied with the finite element method. J Oral Rehabil 2001;28(3):273-279.

39. Willard VP, Kalpakci KN, Reimer AJ, Athanasiou KA. The regional contribution of glycosaminoglycans to temporomandibular joint disc compressive properties. J Biomech Eng 2012;134(1):011011.

40. Eriksson L, Westesson PL, Macher D, Hicks D, Tallents RH. Creation of disc displacement in human temporomandibular joint autopsy specimens. J Oral Maxillofac Surg 1992;50(8):869-873.

41. Westesson PL, Bronstein SL, Liedberg J. Internal derangement of the temporomandibular joint: morphologic description with correlation to joint function. Oral Surg Oral Med Oral Pathol 1985;59(4):323-331.

42. Liu XM, Zhang SY, Yang C, Chen MJ, X YC, Haddad MS, et al. Correlation between disc displacements and locations of disc perforation in the temporomandibular joint.

Dentomaxillofac Radiol 2010;39(3):149-156.

43. Kirk WS, Jr. Morphologic differences between superior and inferior disc surfaces in chronic internal derangement of the temporomandibular joint. J Oral Maxillofac Surg 1990;48(5):455-460.

44. Sun Z, Liu ZJ, Herring SW. Movement of temporomandibular joint tissues during mastication and passive manipulation in miniature pigs. Arch Oral Biol 2002;47(4):293-305. 
TABLE CAPTION

Table 1. Summary of statistical findings for each main and interaction effect.

\begin{tabular}{|l|ccc|ccc|}
\hline & \multicolumn{3}{|c|}{ Main effects } & \multicolumn{3}{c|}{ Interaction Effects } \\
& Deformation & Surface & Region & $\begin{array}{c}\text { Deformation/ } \\
\text { Region }\end{array}$ & $\begin{array}{c}\text { Sefmation/ } \\
\text { Surface }\end{array}$ & $\begin{array}{c}\text { Surface/ } \\
\text { Region }\end{array}$ \\
\hline Ultimate Tensile Strength & $\mathbf{D}<\mathbf{N}, \mathbf{p}<\mathbf{0 . 0 0 1}$ & $\mathbf{l} \mathbf{S}, \mathbf{p}=\mathbf{0 . 0 2 3}$ & $\mathbf{p}<\mathbf{0 . 0 0 1}$ & $\mathbf{p}<\mathbf{0 . 0 0 7}$ & $\mathbf{p}=\mathbf{0 . 0 4 9}$ & $\mathrm{p}=0.281$ \\
Tensile Youngs Modulus & $\mathbf{D}<\mathbf{N}, \mathbf{p}<\mathbf{0 . 0 0 1}$ & $\mathbf{l} \mathbf{S}, \mathbf{p}=\mathbf{0 . 0 2 0}$ & $\mathbf{p}<\mathbf{0 . 0 0 1}$ & $\mathbf{p}<\mathbf{0 . 0 0 1}$ & $\mathbf{p}=0.058$ & $\mathbf{p}=0.384$ \\
Strain at Failure & $\mathbf{D} \mathbf{N}, \mathbf{p}=\mathbf{0 . 0 1 9}$ & $\mathbf{p}=0.289$ & $\mathbf{p}<\mathbf{0 . 0 0 1}$ & $\mathbf{p}=0.272$ & $\mathbf{p}=0.504$ & $\mathbf{p}=0.526$ \\
Compressive Modulus & $\mathbf{D}>\mathbf{N}, \mathbf{p}=\mathbf{0 . 0 0 5}$ & $\mathbf{p}=0.126$ & $\mathbf{p}<\mathbf{0 . 0 2 4}$ & $\mathbf{p}=\mathbf{0 . 0 1 6}$ & $\mathbf{p}=0.679$ & $\mathbf{p}=\mathbf{0 . 0 2 8}$ \\
Cell Density & $\mathbf{D} \mathbf{N}, \mathbf{p}<\mathbf{0 . 0 0 1}$ & $\mathbf{p}=0.988$ & $\mathbf{p}<\mathbf{0 . 0 0 1}$ & $\mathbf{p}=0.057$ & $\mathbf{p}=0.981$ & $\mathbf{p}=0.598$ \\
sGAG content & $\mathbf{p}=0.077$ & $\mathbf{p}=0.179$ & $\mathbf{p}<\mathbf{0 . 0 0 1}$ & $\mathbf{p}=0.079$ & $\mathbf{p}=0.745$ & $\mathbf{p}=0.970$ \\
\hline
\end{tabular}

FIGURE CAPTIONS

Figure 1. Disc testing regions. Lateral (L), posterior (P), intermediate zone (IZ), anterior (A), and medial (M) regions were evaluated during testing. Sections were further divided into superior (S) and inferior (I) samples. Rectangular sections were used for tensile biomechanical evaluation, while circular regions were used compressive biomechanical and biochemical analysis. Histology was performed on remaining untested tissue regions.

Figure 2. Macroscopic comparison of morphologically normal and deformed pTMJ discs. A) Superior surface, sagittal cross section, and inferior surface gross morphology of normal and deformed pTMJ discs. B) Average medial-lateral (M-L) and anterior-posterior (A-P) measurement did not vary between normal $(n=7)$ and deformed $(n=4)$ groups. C) Deformed pTMJ discs showed significant thickening in the posterior band (PB), intermediate zone (IZ), and anterior band $(\mathrm{AB})$ denoted with an $*(\mathrm{p}<0.05)$.

Figure 3. Regional tensile biomechanical properties. The inferior (I) and superior (S) surfaces were evaluated in the posterior (P), lateral (L), intermediate zone (IZ), medial (M), and anterior (A) regions. Deformed disc showed statistically significant decrease in A) ultimate tensile strength (UTS) and B) Young's Modulus, and increase in C) elongation at failure. Regional variations detected by three way ANOVA are denoted by not sharing a letter indicating significant difference in post-hoc testing $(\mathrm{p}<0.005)$. Significant region-deformation differences are denoted with \#.

Figure 4. Regional compressive biomechanical properties. The inferior (I) and superior (S) surfaces were evaluated in the posterior (P), lateral (L), intermediate zone (IZ), medial (M), and anterior (A) regions. Deformed discs had a statistically significant increase in compressive modulus. Regional variations were not significant in post-hoc testing. Significant regiondeformation differences are denoted with \# $(\mathrm{p}<0.01)$ and significant surface-regional variations are denoted with * $(\mathrm{p}<0.02)$.

Figure 5. Regional cellular and sGAG quantification. The inferior (I) and superior (S) surfaces were evaluated in the posterior (P), lateral (L), intermediate zone (IZ), medial (M), and anterior (A) regions. A) Deformed discs had higher cell density compared to morphologically normal discs and regional variations were detected. B) There were no significant differences in sGAG content between deformed and normal discs, but significant regional variations were 
present. Regional variations detected by three way ANOVA are denoted by not sharing a letter indicating significant difference in post-hoc testing $(\mathrm{p}<0.005)$.

Figure 6. Extracellular matrix alignment in the intermediate zone of the pTMJ discs. H\&E staining shows increase in cell density and loss of characteristic anterior posterior fiber alignment in the intermediate zone of the deformed pTMJ discs. Disc cross section schematics indicates orientation of the micrographs. Scale bars indicates $100 \mu \mathrm{m}$

Figure 7. Formation of cellular nodules on the inferior disc surface. Hyperplasia and cell dense nodules on inferior surface of discs. Scale bars indicates $200 \mu \mathrm{m}$. 


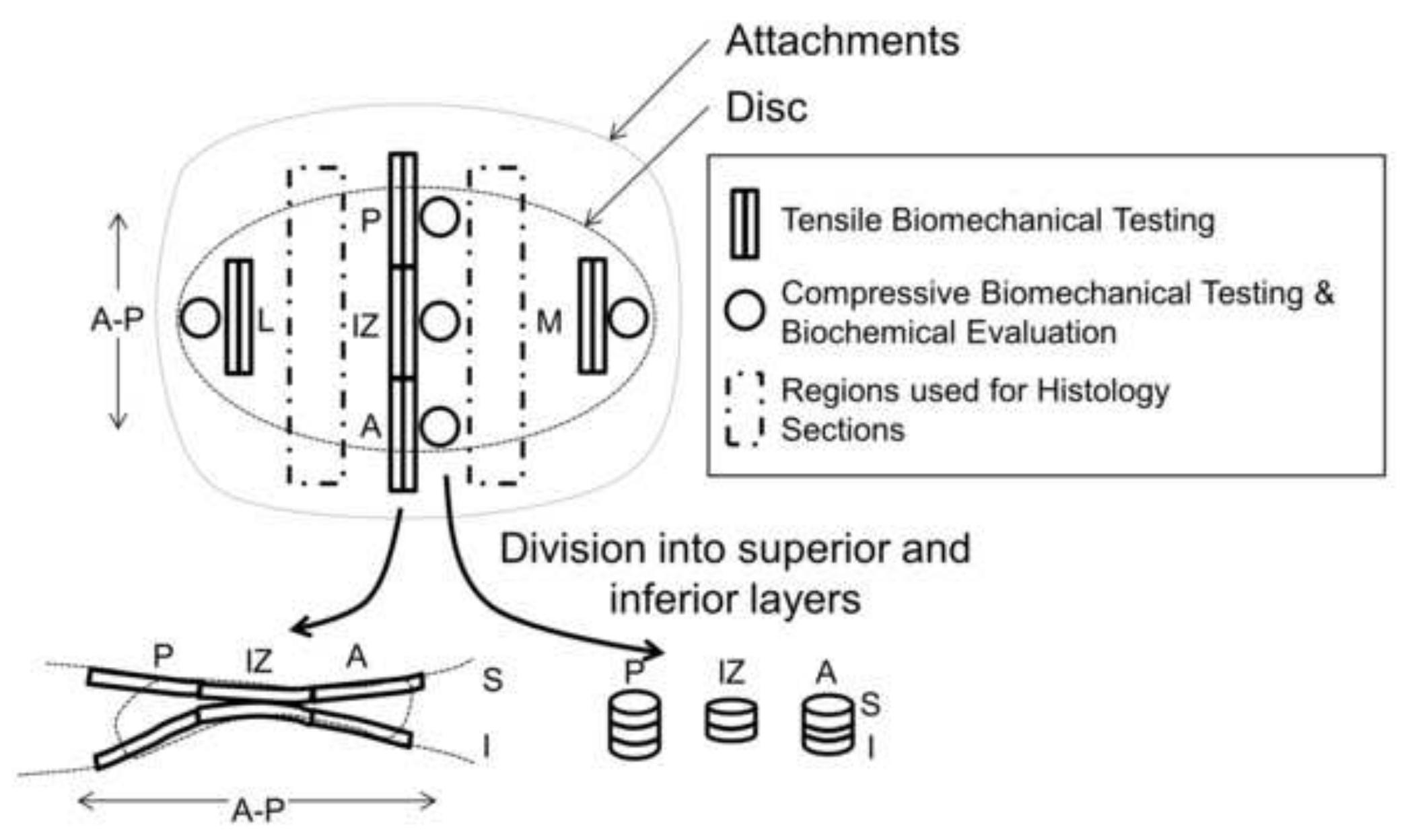




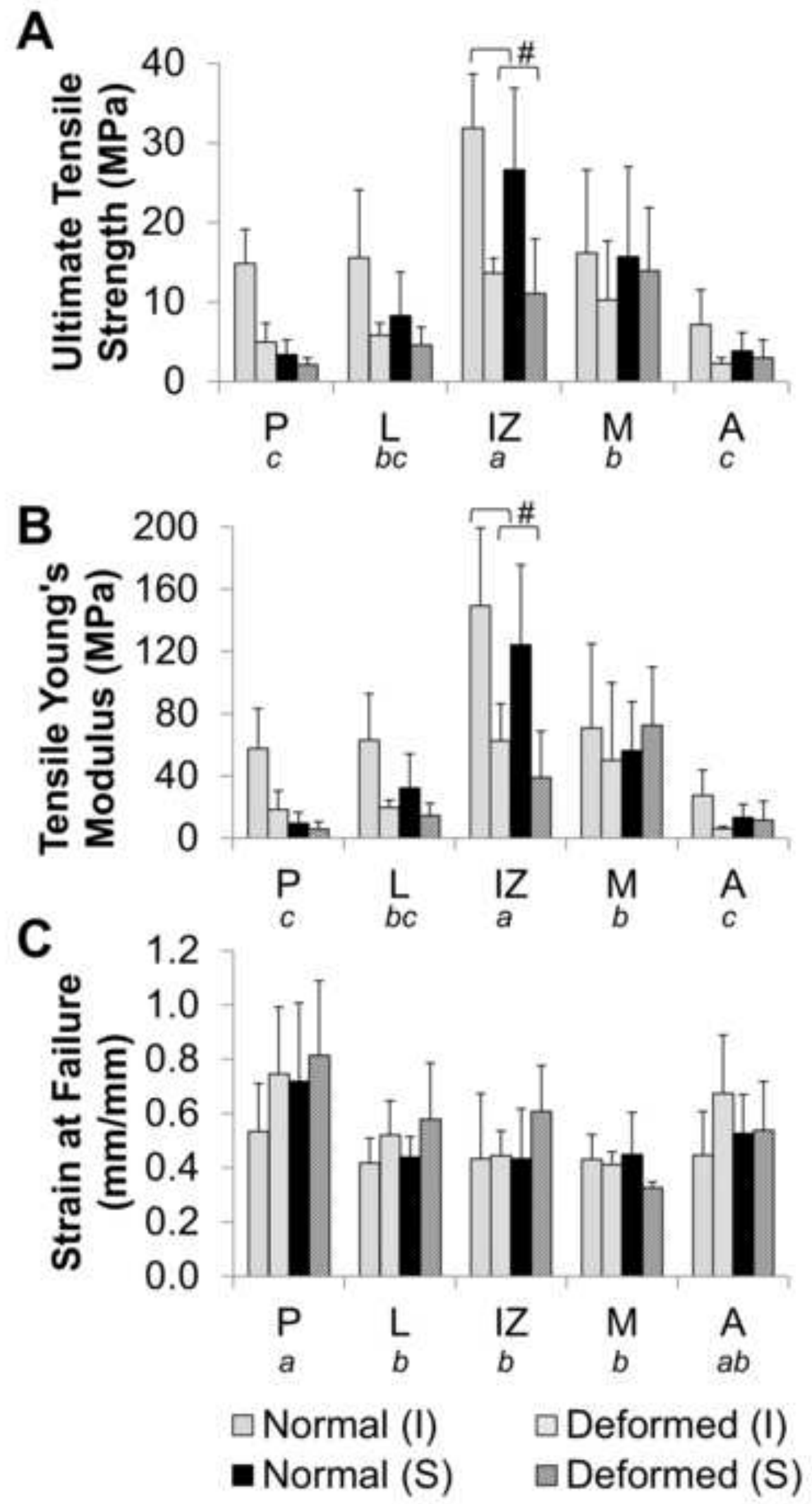


Figure4

Click here to download high resolution image

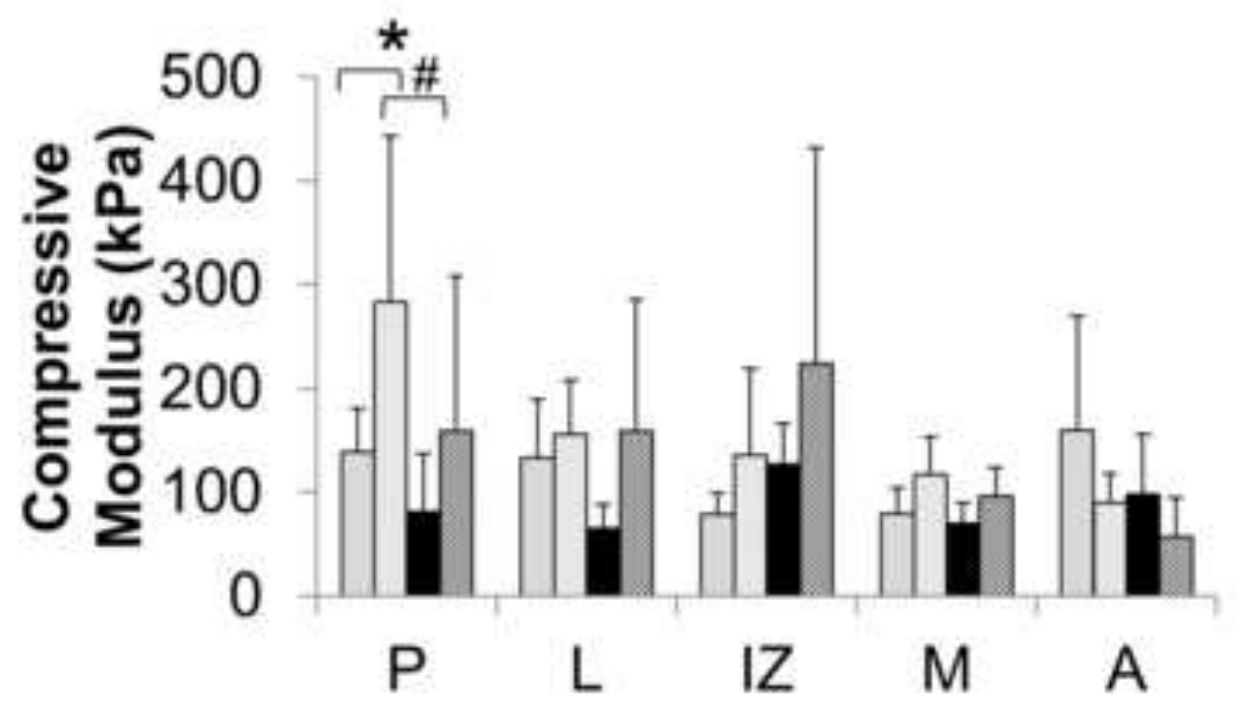




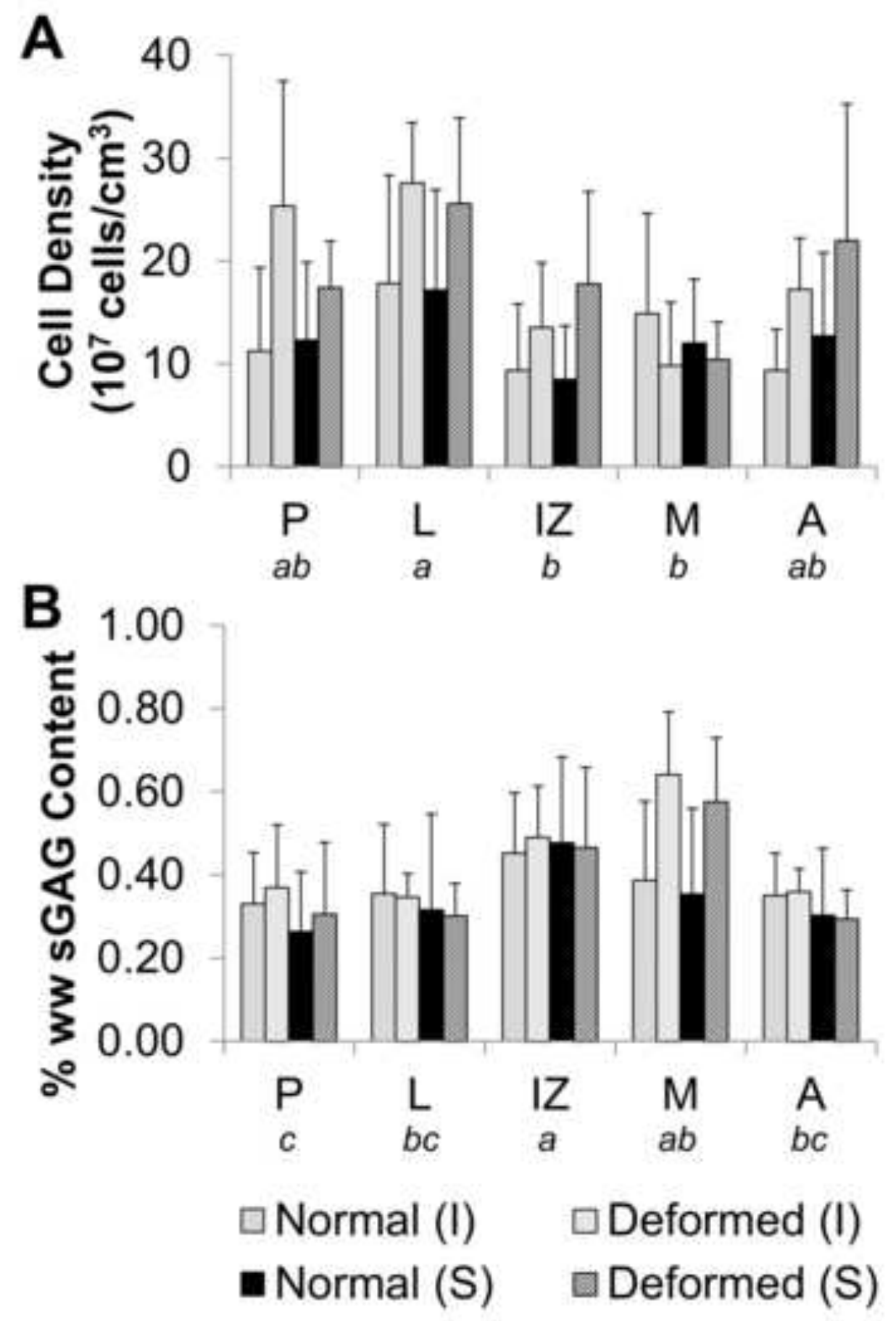




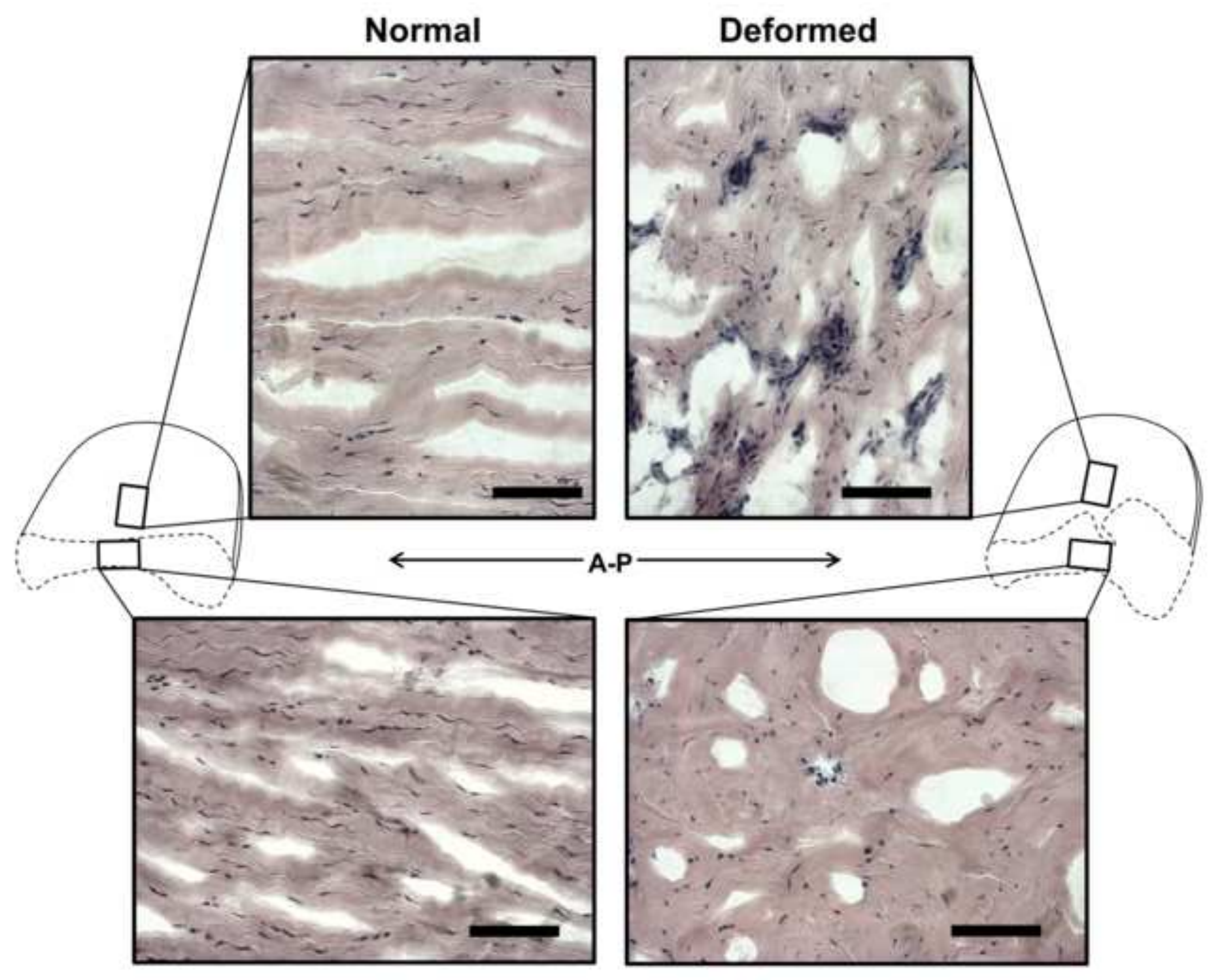


